\title{
Control of Meniscus Stability in Medium Thickness-straight Walls Slab Mould
}

\author{
Yeraldi Sujei GUTIERREZ-MONTIEL ${ }^{1)}$ and Rodolfo D. MORALES ${ }^{2 *}$
}

1) Graduate Student at Instituto Politecnico Nacional-ESIQIE, Department of Metallurgy and Materials Engineering, Ed. 7 UPALM, Col. Zacatenco, Mexico D.F. C.P. $07338 . \quad 2)$ 2) Professor at Instituto Politecnico Nacional-ESIQIE, Department of Metallurgy and Materials Engineering, Ed. 7 UPALM, Col. Zacatenco, Mexico D.F. C.P. 07338.

(Received on June 20, 2012; accepted on October 29, 2012)

\begin{abstract}
Meniscus stability influenced by fluid flow patterns inside a medium thickness- parallel mould walls was analyzed using water modeling techniques including dye tracer injection, PIV and ultrasonic measurements on real time of meniscus levels. Two different submerged entry nozzles (SEN) were compared, the first is under current operation at the caster which yields a very high level of turbulence and the second is a computer aided design SEN which is aimed to reemplace the first one. The experimental results indicate that the first SEN 1 induces a single-unstable large flow roll which promotes large bath oscillations and generation of vortex flows on the bath surface. Fluid speeds at meniscus level reach magnitudes as large as $0.6 \mathrm{~m} / \mathrm{min}$ and in the wall mould as high as a $0.95 \mathrm{~m} / \mathrm{s}$ which are large enough to attain "shell washing conditions". Meanwhile SEN 2, new design, yields a very stable meniscus with speeds as large as $0.45 \mathrm{~m} / \mathrm{s}$ at the meniscus in a stable-double flow roll. The analysis of entrapment and entrainment mechanisms of slag leads to conclude that the first SEN will induce slag entrapment under almost all operating conditions while the second SEN will induce slag entrapment only under very specific conditions of high casting speed of $3.68 \mathrm{~m} / \mathrm{min}$.
\end{abstract}

KEY WORDS: slab mould; flux entrapment; PIV; meniscus levels; SEN design.

\section{Introduction}

Conventional thick slab moulds $(200-300 \mathrm{~mm})$ operate at casting speeds that go from 0.8 to $2.5 \mathrm{~m} / \mathrm{min}^{1)}$ depending on the specific machine design and local constraints at the caster. In the upper bounds of casting speed the employment of electromagnetic braking (EMBr) or a suitable SEN design are strongly recommended since the level of turbulence in the mould deforms the meniscus of the metal to the extent of making possible flux overlapping and slag entrainment well inside the mould. Medium Thickness-Straight and parallel Wide Walls Moulds (MTSWM) (90-100 mm) are definitively designed to operate at higher casting speeds than conventional thick slab moulds. Casting speeds in these casters exceed $3 \mathrm{~m} / \mathrm{min}$ and therefore, turbulence intensity is considerably higher than in conventional thick slabs owing to higher steel throughputs and more confined spaces which makes inevitably, in principle, the implementation of suitable SEN designs.

It is worthy to underline that high casting speeds promote large melt velocities in the meniscus which give origin to subsurface mould slag entrainment, particularly in ultralow carbon steels. ${ }^{1-3)}$ Some mechanisms have been reported regarding mould flux entrainment by the melt. ${ }^{4)}$ The main mechanisms of mould slag entrainment are meniscus freezing and hook formation, Von Karman vortex formation, slag crawling, meniscus level fluctuations, shear layer instability, meniscus standing wave instability, top surface balding and upward flow impinging on meniscus. All those possible slag

\footnotetext{
* Corresponding author: E-mail: ketechnologies@prodigy.net.mx DOI: http://dx.doi.org/10.2355/isijinternational.53.230
}

entrainment mechanisms are intimately related with fluid flow pattern of steel in the mould. Given the importance of fluid flow control in continuous casting researchers have focused their efforts in understanding flow turbulence in moulds. Ramos-Banderas et al. ${ }^{5}$ analyzed, through Large Eddy Simulation and physical modelling, the transient nature of steel flow during steel discharge from a nozzle in a conventional slab. Radical changes of fluid flow patterns within short periods of time were identified demonstrating that there is not a steady fluid flow pattern. These findings are on line with the former results of Gupta et al. ${ }^{6}$ who reported asymmetrical flows in the mould and unsteady flow conditions. Honeyhands and Hebertson ${ }^{7)}$ explained qualitatively those instable flows through the principle of the Strouhal number and the replacement ratio of the fluid volumetric flow and the volume of the melt in the mould. A good correlation was obtained between the frequency of meniscus oscillations and the replacement ratio. However, more than a fundamental explanation this contribution makes a valuable practical approach to estimate approximate fluid flow effects. Fundamental explanations about flow turbulence, in thin slabs moulds have been provided in a series of papers by Torres-Alonso et al. ${ }^{8-10)}$ They found, through extensive physical modelling and mathematical simulations, the relationship between low frequency-large scale meniscus oscillations and the imbalance of the turbulent kinetic energy. This imbalance is originated by the shearing between the discharging jets and the surrounding fluid in the mould. In others works by the author's Group ${ }^{11,12)}$ fluid flows of steel under the effects of flow separation in the ports and the associated back flow as well as bath surface topography were analyzed through mathemati- 
Table 1. Mould operating conditions.

\begin{tabular}{cccccc}
\hline TEST & $\begin{array}{c}\text { Width } \\
(\mathrm{mm})\end{array}$ & $\begin{array}{c}\text { Thickness } \\
(\mathrm{mm})\end{array}$ & $\begin{array}{c}\text { Casting } \\
\text { Speed } \\
(\mathrm{m} / \mathrm{min})\end{array}$ & $\begin{array}{c}\text { Flow Rate } \\
(\mathrm{l} / \mathrm{s})\end{array}$ & $\begin{array}{c}\text { Submergence } \\
\text { Depth }(\mathrm{mm})\end{array}$ \\
\hline 1 & 1270 & 90 & 3.30 & 6.7 & 125 \\
2 & 1270 & 90 & 3.68 & 7.5 & 125 \\
\hline
\end{tabular}

cal simulation and fluid flow experimental techniques. Those researches were focused on the turbulence problem in thin slab moulds where casting speeds are as high as $7 \mathrm{~m} / \mathrm{min}$.

The present research deals with steel flow in the MTSWM of a caster belonging to an USA company. The aim is to substitute the current SEN, which induces unusual high levels of turbulence and meniscus instabilities, by a new design that must provide enhanced fluid flow patterns. For this purpose the flow is deeply analyzed through physical modelling approaches. Mould dimensions and casting operating conditions are reported in Table 1. The challenge faced here is to come out with a new SEN design, different to the one under current usage, which must deliver steel in this mould maintaining meniscus stability and slab quality. Next lines explain the details of this development.

\section{Experimental Procedure}

A full scale model of the actual mould $\left(1270 \times 90 \mathrm{~mm}^{2}\right)$ was manufactured from transparent plastic. The mould model was inserted into a pit full of water to represent the continuity of the strand. Water is elevated through an immersed pump to a full scale tundish fixing the bath height at the same magnitude as in the actual tundish. In the pipe used to elevate the water a flow meter and a precision valve were inserted to be able to measure continuously the amount of water fed into the mould. The flow rate from the tundish to the mould was controlled through a rod whose tip bears a machined plug with the same geometry of the actual stopper rod made of plastic. The discharge of water into the mould model was performed using the actual two-port SEN under current usage at the caster. The new design of the SEN was previously designed through CFD-mathematical simulations. Once having the final design a plastic version was manufactured to be employed in the experimental work to make comparisons with the current SEN. The experimental techniques employed to study the flow in the mould are described in the next paragraphs.

Three ultrasonic water level sensors were positioned, at each side of the SEN (six in total) to follow the meniscus oscillations in real time. The signals of these sensors were converted into digital data using an acquisition card with a capacity of receiving 12 simultaneous analogical signals at frequencies as high as $10 \mathrm{KHz}$. Digital data were received by a plotter system to visualize meniscus oscillations during the experimental work and were recorded for further analysis. Water 2D velocity fields were also recorded using Particle Image Velocimeter (PIV) techniques. The experimental details of the PIV recordings can be found in Ref. 13). This experimental tool permitted the recording of the unsteady fluid flows in real time. Other derived flow variables included vorticity fields, streamlines, etc. Finally, injection of a red dye tracer in the upper part of the SEN was used to visualize, through conventional video techniques, the fluid flow patterns and meniscus oscillations. Figure 1 shows a photo of the full experimental setup described here. Figure 2 shows schemes of the current SEN 1 (two ports) and the computer aided design SEN 2 (five ports and two orifices). In order to compare directly the performance of both SEN's only one casting mould dimension (mentioned above) was considered in the present study, casting speeds

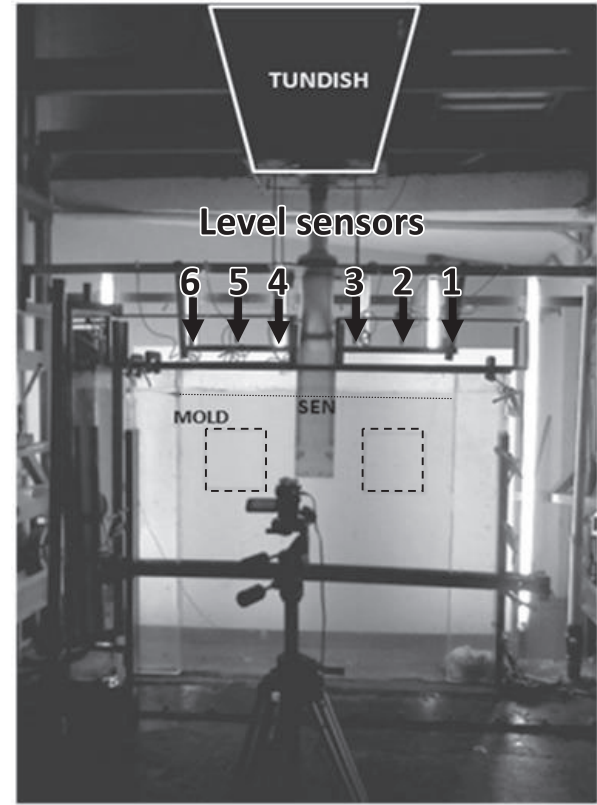

Fig. 1. Experimental setup.

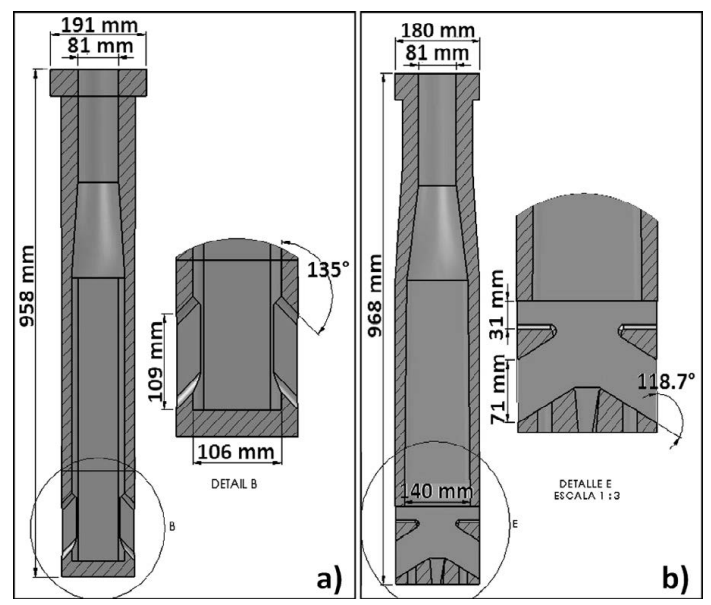

Fig. 2. Geometric characteristics of the original a) SEN 1 and new (b) SEN 2 submerged entry nozzles.

employed in this caster are 3.30 and $3.68 \mathrm{~m} /$ minute $(2.81$ and 3.15 tons/minute respectively), a SEN depth of $125 \mathrm{~mm}$ (just as is the actual caster) measured from the bath level to the upper edge of the lateral ports. This immersion depth of the SEN is the current shallowest position used in this caster.

\section{Results and Discussion}

\subsection{Meniscus Levels}

Figure 3 shows a direct comparison of the meniscus stability using SEN's 1 and 2 at a casting speed of $3.30 \mathrm{~m} /$ minute. The printed squares in the mould broad face indicate the position and the size of the EMBr system in the actual caster, while the lines in the jets are used to estimate parameters like spread angles of the jets, jet angle and impinging point in the narrow mould faces. The dimensions in the right narrow mould faces indicate the length of the impinging points of the jet on the narrow mould faces. Under those conditions the location of the impinging jet points are very close to each other. As seen here, SEN 1 (Figs. 4(a)-4(f)) induces a strong meniscus deformation at half the way between the SEN wall and the narrow faces. That is not the case using SEN 2 since the meniscus remains stable and independent 
of time as can be seen in Figs. 4(g)-4(1)where the meniscus levels are recorded at different times. Considering SEN 1 again it is observed that there is the development of high standing waves at times between 1 and 6 seconds. In Fig. 4(a) the standing wave is travelling from the left narrow face and 2 seconds later it develops from the right narrow face. Three seconds later (Fig. 4(c)) the waves start a new oscillation cycle from the left narrow face. These cyclic oscillations remain operating until a time of 20 seconds that is when the meniscus reaches an apparent flatness (Fig. 4(d)). This condition lasts 10 seconds approximately and later the
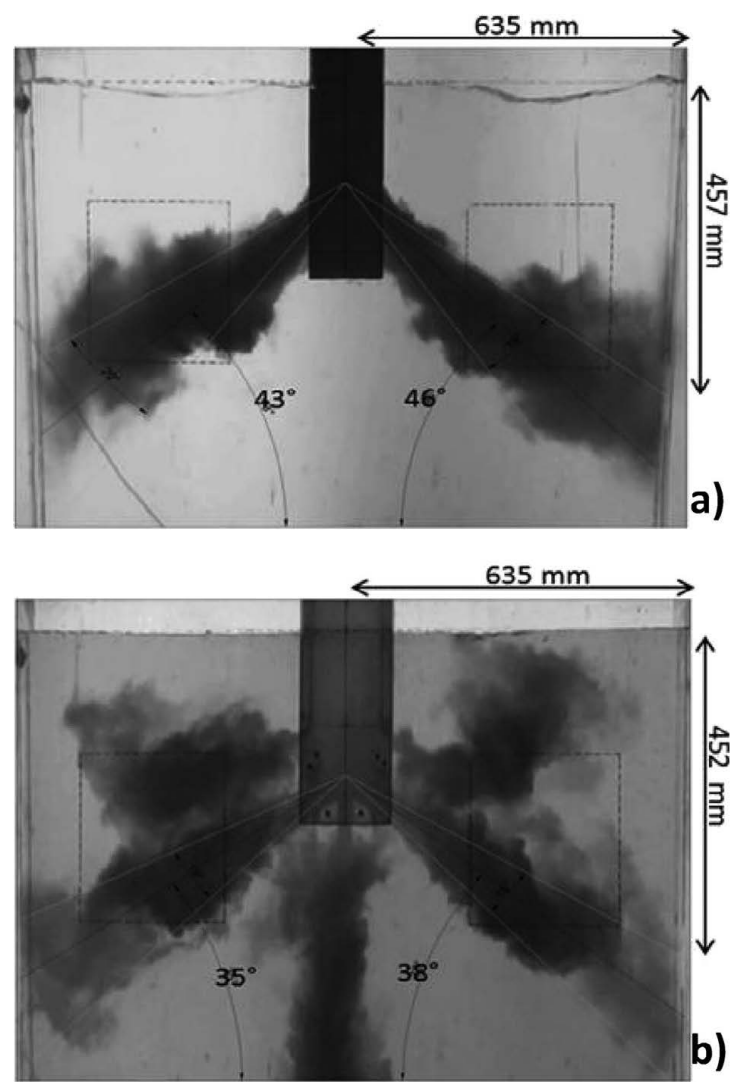

Fig. 3. Jet characteristics using a) SEN 1 and b) SEN 2 at casting speed of $3.30 \mathrm{~m} / \mathrm{min}$. Lengths in the left sides of both figures indicate the impinging point of the discharging jets. cyclic oscillation pattern comes back to repeat the development of the standing waves. Figures 5(a)-5(1) shows the corresponding meniscus profiles for both SEN's at a casting speed of $3.68 \mathrm{~m} / \mathrm{min}$. Standing waves using SEN 1 observes a similar behaviour to that described above, however, depressions are deeper and the apparent fluid velocity is higher. Since the casting speed is higher, turbulence is considerably increased and the periods of flat meniscus are considerably shortened. SEN 2, on the other hand, yields stable meniscus with very shallow depressions of standing waves indicating, qualitatively, that the flow is less turbulent. These initial results demonstrate one important aspect; there is a complete justification to change design of SEN 1 by another one with improved performance.

Meniscus dynamics, (measured through the instantaneous meniscus levels by the ultrasonic sensors) for a casting speed of $3.30 \mathrm{~m} / \mathrm{min}$ is shown in Figs. 6(a)-6(c) for each pair of sensors located at the same positions at each side of the SEN 1. Data in Fig. 6(a) indicates that most of the oscillations in the mould corners (sensors 1 and 6) are positive. These are effects originated by the upper roll flows coming from the narrow faces to the SEN position which induce an uprising motion on the bath surface at the mould corner. Both flows seem to work uncoupled since both jets deliver the fluid with different momentum transfer at a same given instant. Meniscus levels detected by sensors 2 and 5, Fig. 6(b) (located at half the way between the narrow faces and the SEN) are mostly negative due to the persistent formation of a depression which takes place due to the effects of the upper roll flows. Meniscus levels close to the SEN, Fig. 6(c), (sensors 3 and 4) report slightly positive magnitudes of uprising motion owing to the braking effects of the close SEN on fluid motion. Figures 7(a)-7(c) show the corresponding meniscus levels using SEN 2 at the same casting speed. As can be seen in those figures, meniscus levels for all positions are considerably smaller than in the precedent case. The oscillations in each sensor are so similar among them that it is difficult to distinguish the differences between the recordings of each pair of sensors. Therefore, it can be deduced that in this case the upper rolls reach the meniscus simultaneously avoiding excessive wave's amplitudes.

Meniscus levels at a casting speed of $3.68 \mathrm{~m} / \mathrm{min}$ using SEN 1 are shown in Figs. 8(a)-8(c) for each pair of sensors 1-6, 2-5 and 3-4 respectively allowing comparisons among the same positions at both sides of the SEN 1. As is seen, sensors 1 and 6 (near mould corners) indicate that the bath

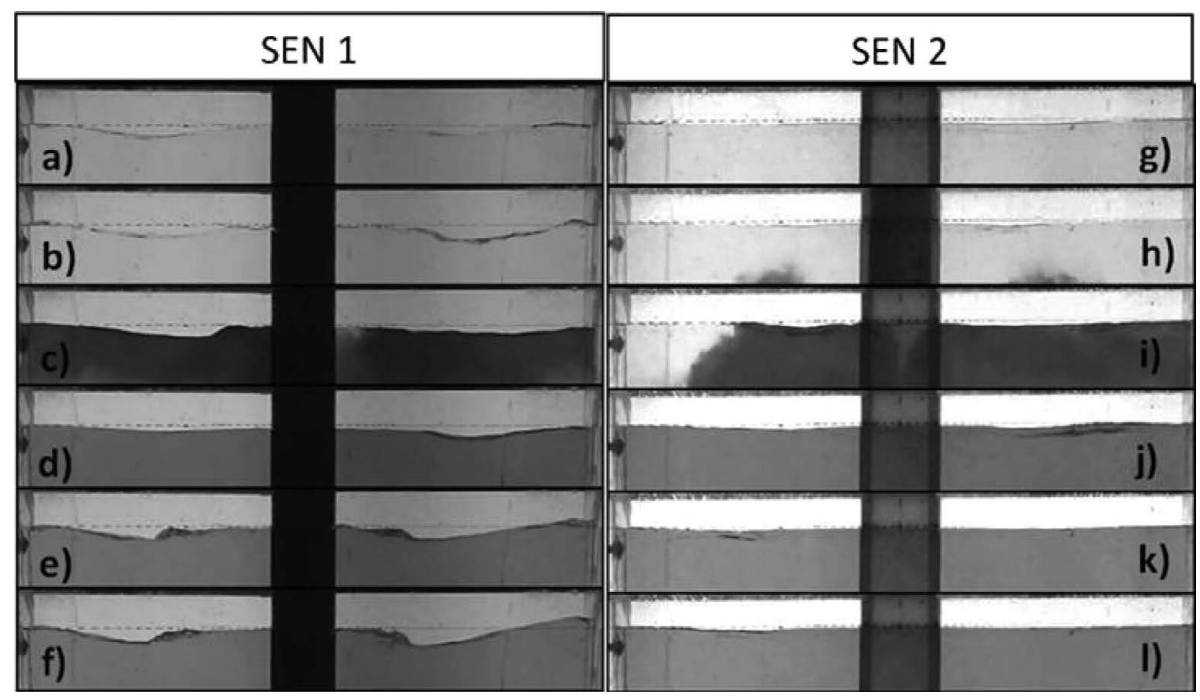

Fig. 4. Meniscus profiles at diferent times at a casting speed of $3.30 \mathrm{~m} / \mathrm{min}$. a) $1 \mathrm{~s} \mathrm{~b}) 3 \mathrm{~s} \mathrm{c}$ ) $6 \mathrm{~s} \mathrm{~d}) 20 \mathrm{~s} \mathrm{e}) 30 \mathrm{~s} \mathrm{f}) 31 \mathrm{~s} \mathrm{~g}) 1 \mathrm{~s}$ h) $3 \mathrm{si}$ i) $6 \mathrm{sj}$ j) $20 \mathrm{sk}$ ) $30 \mathrm{~s}$ l) $31 \mathrm{~s}$. 


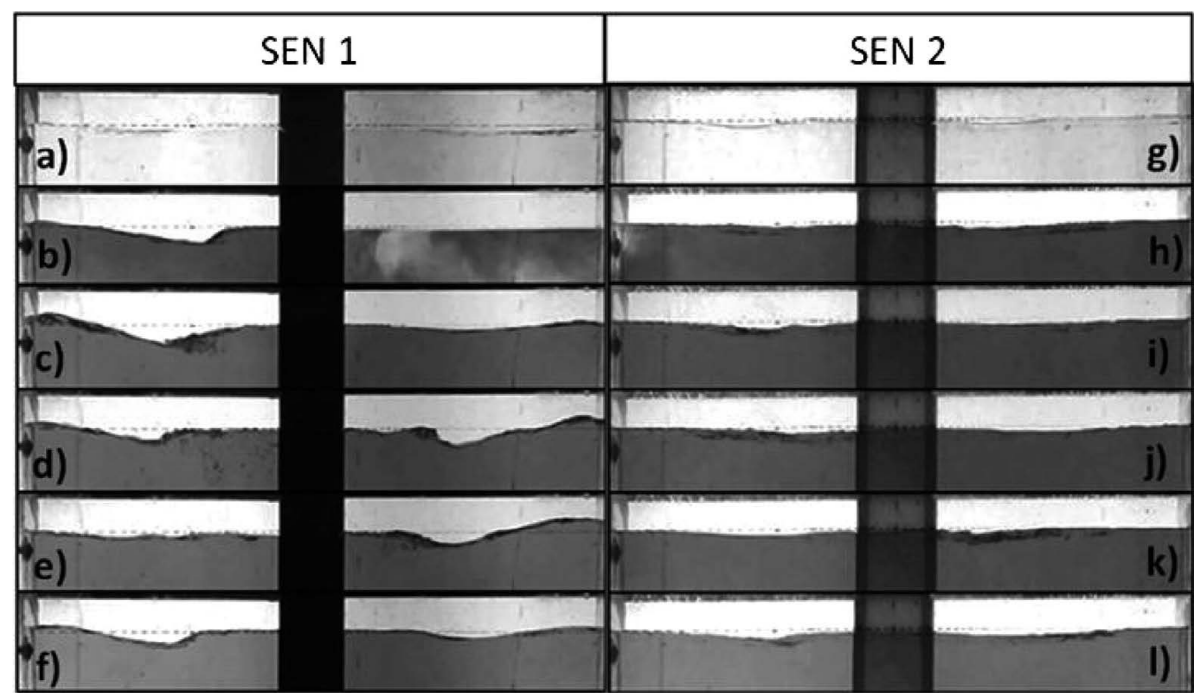

Fig. 5. Meniscus profiles at diferent times at a casting speed of $3.68 \mathrm{~m} / \mathrm{min}$. a) $1 \mathrm{~s} \mathrm{~b}) 3 \mathrm{~s} \mathrm{c}) 6 \mathrm{sd}$ ) $20 \mathrm{~s} \mathrm{e}) 30 \mathrm{~s} \mathrm{f}) 31 \mathrm{~s} \mathrm{~g}) 1 \mathrm{~s}$ h) $3 \mathrm{~s}$ i) $6 \mathrm{sj}$ ) $20 \mathrm{sk}$ ) $30 \mathrm{~s}$ l) $31 \mathrm{~s}$.

SEN 1

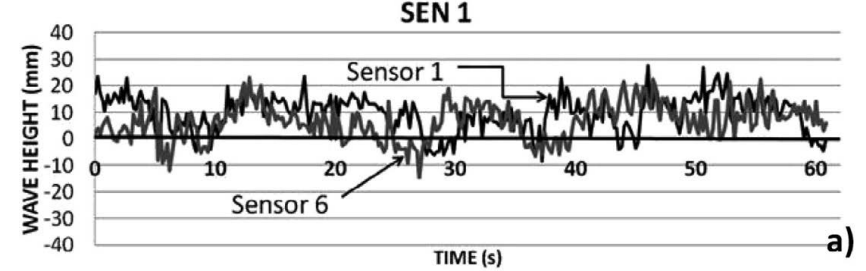

SEN 1

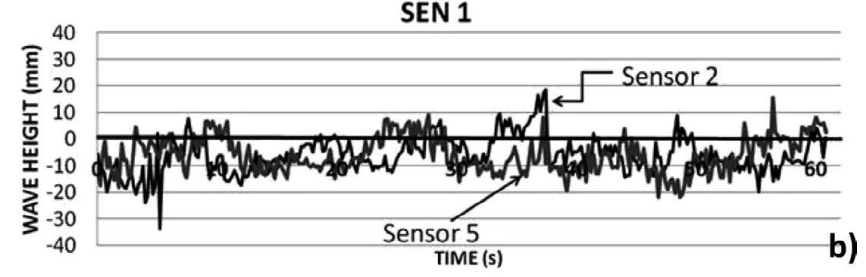

SEN 1

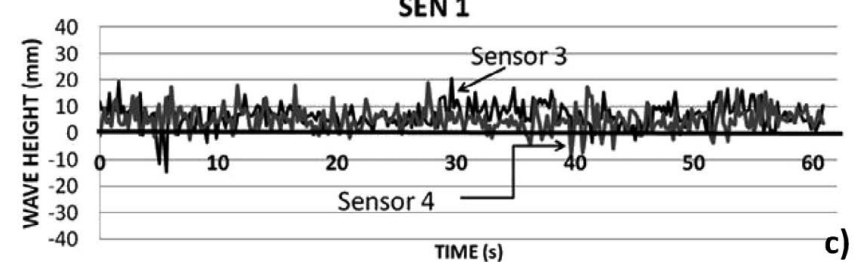

Fig. 6. Variations of meniscus levels with casting time at a casting speed of $3.30 \mathrm{~m} / \mathrm{min}$ using SEN 1. a) Mould corner. b) $1 / 4$ mould width. c) Near SEN.

oscillates considerably since when sensor 1 indicates the presence of a wave hill sensor 6 indicates, at the same time, a lower level keeping, most of the time, positive magnitudes. This is a signal that the higher fluid throughputs increase the decoupling pattern of the discharging jets. Hereby, when the left upper roll is reaching the meniscus the right flow is still on its way promoting heavy bath oscillations. Same observations are applicable to sensors 2 and 5 (located at half the way between the mould corner and the SEN wall), although, the amplitude of the bath oscillations exceed $30 \mathrm{~mm}$ above and below the static meniscus level. Oscillations are mostly negative due to the persistent existence of the depression on the bath surface. Sensors 3 and 4 (located close to the SEN wall) yield more coupled and smaller oscillations probably due to the mitigating effects of the close SEN wall. Figures 9(a)-9(c) show meniscus dynamics for the same casting speed using SEN 2 corre-
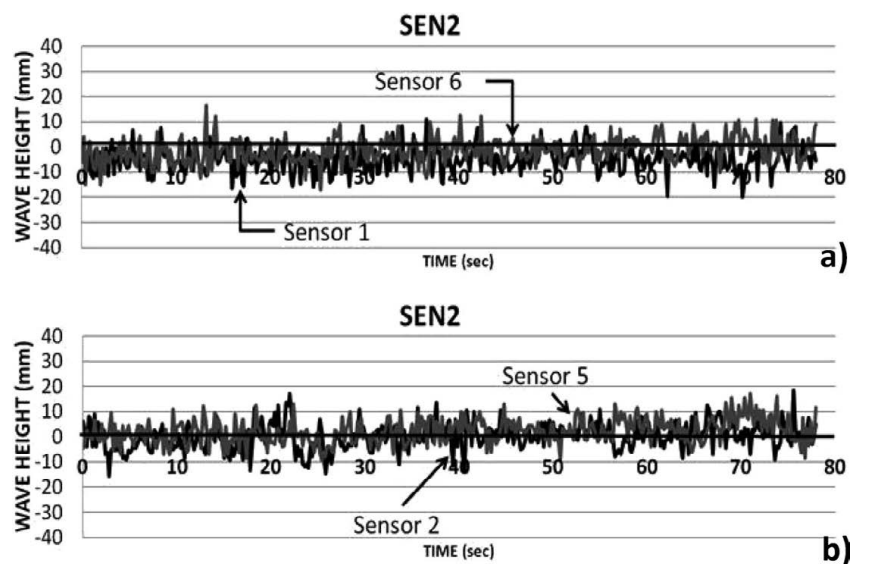

SEN2

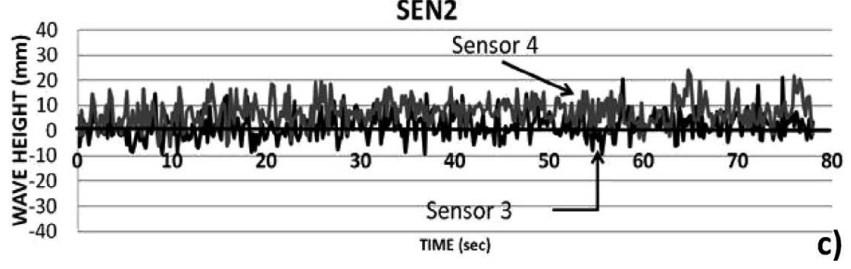

Fig. 7. Variations of meniscus levels with casting time at a casting speed of $3.30 \mathrm{~m} / \mathrm{min}$ using SEN 2. a) Mould corner. b) $1 / 4$ mould width. c) Near SEN.

sponding to pair of sensors 1-6, 2-5 and 3-4, respectively. Generally speaking, the meniscus level maintains a considerably higher stability than in the precedent case with wave amplitudes going from -10 to $+10 \mathrm{~mm}$. The small level oscillations in the mould corners mean that the upper roll flows have smaller velocities which must be corroborated later on with by PIV measurements. Sensors 2 and 5 show, clearly, the inexistence of the bath surface depression while sensors 3 and 4 indicate slightly larger oscillations than in the case of SEN 1 at the same positions.

Standard deviations of meniscus level data for each sensor are presented in Figs. 10(a) and 10(b) for 3.3 and 3.68 $\mathrm{m} /$ minute respectively for both SEN's. SEN 2 yields even standard deviations along the mould width independently of the casting speed meaning meniscus variations fixed between certain limits. However, SEN 1 reports very large standard deviations along the mould width and especially in 


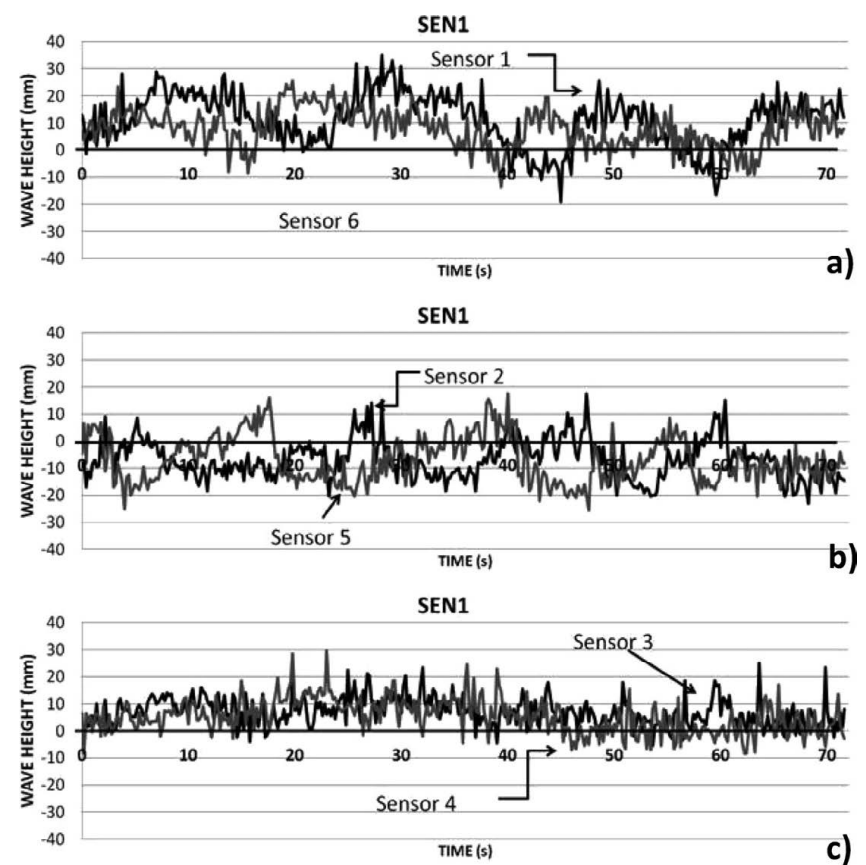

Fig. 8. Variations of meniscus levels with casting time at a casting speed of $3.68 \mathrm{~m} / \mathrm{min}$ using SEN 1. a) Mould corner. b) $1 / 4$ mould width. c) Near SEN
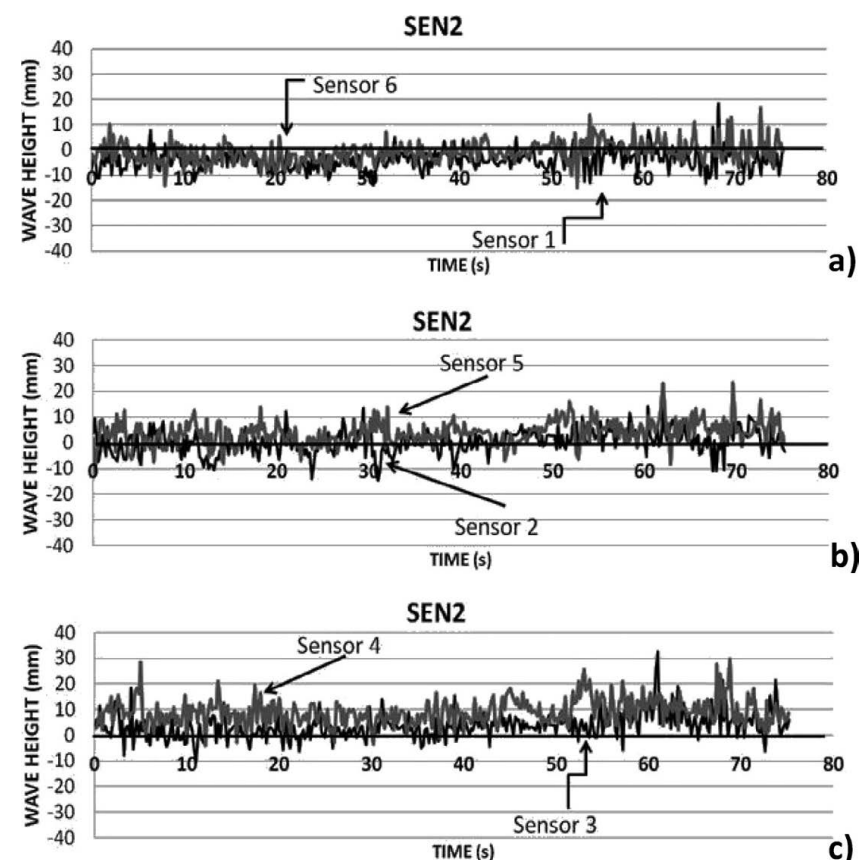

Fig. 9. Variations of meniscus levels with casting time at a casting speed of $3.68 \mathrm{~m} / \mathrm{min}$ using SEN 2. a) Mould corner. b) $1 / 4$ mould width. c) Near SEN.

the mould corners very large and strong oscillations of the whole bath is again evident. Besides, the standard deviations in the mould corners, using SEN 1, indicates a biased flow which remains for long periods of time making evident a permanent unstable fluid flow pattern (not double roll flow neither single roll flow).

\subsection{PIV Measurements}

Instantaneous velocity fields at a casting speed of 3.3 $\mathrm{m} /$ minute for using SEN 1 are shown in Figs. 11(a)-11(c) and velocity fields using SEN 2 are shown in Figs. 11(d)-11(f). Some observations derived from these fields are as follows: a)

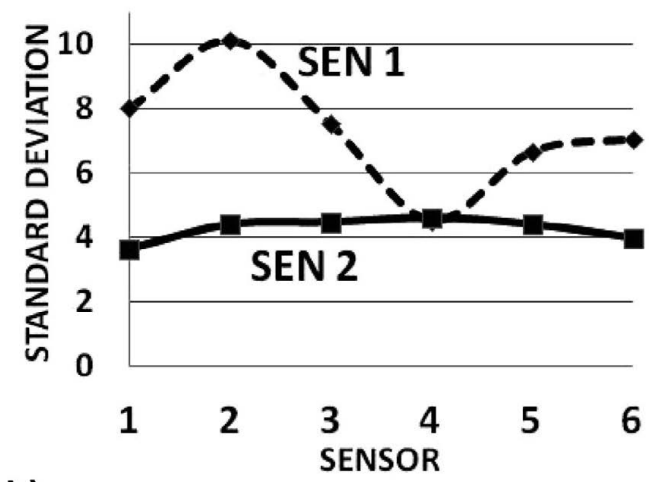

b)

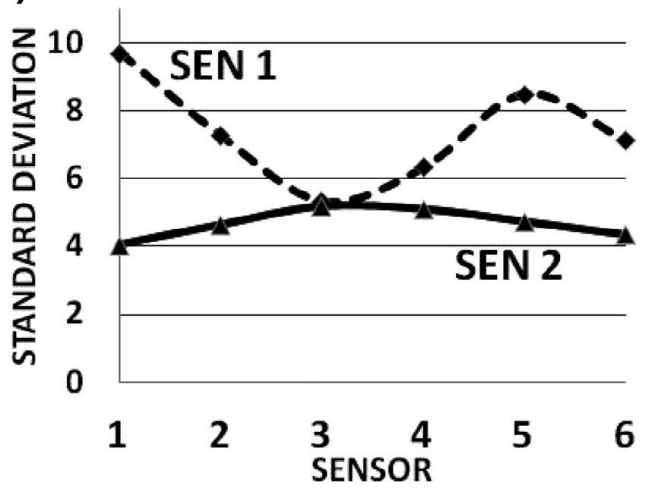

Fig. 10. Standard deviations of meniscus levels for each sensor using SEN's 1 and 2. Casting speeds a) $3.30 \mathrm{~m} / \mathrm{min}$ and b) $3.68 \mathrm{~m} / \mathrm{min}$.

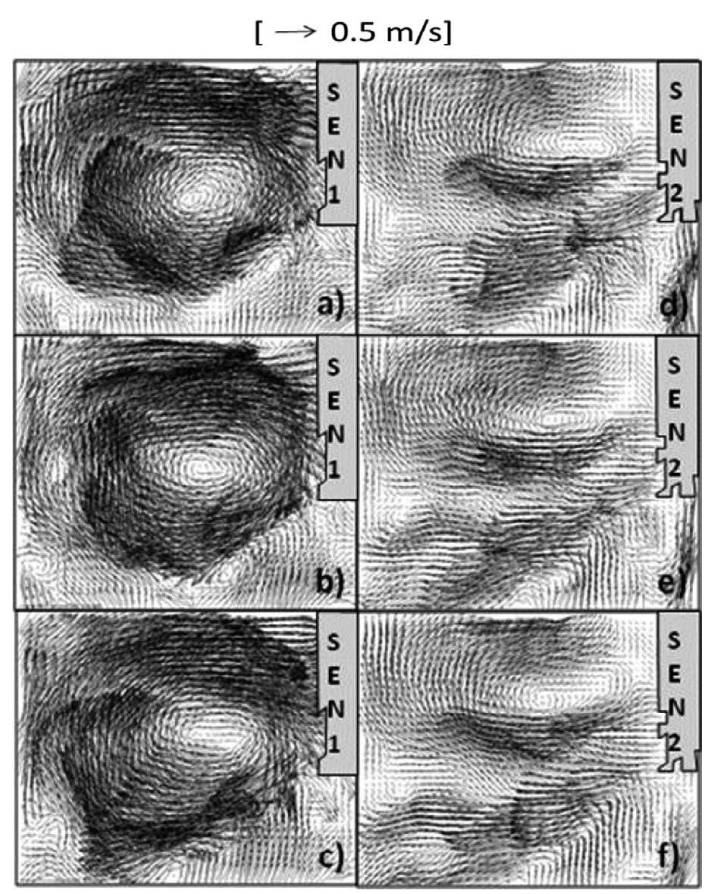

Fig. 11. Velocity fields in the central plane of the mould using a-c) SEN 1 and d-f) SEN 2 at a casting speed of $3.30 \mathrm{~m} / \mathrm{min}$. Time difference among each image is $0.25 \mathrm{~s}$.

- Fluid flow using SEN 1 (Figs. 11(a)-11(c)) consists of a large single roll which is the origin of the highly instable meniscus. The fluid leaves the SEN and flows downwards to rotate later and flowing toward the SEN at the meniscus level.

- Fluid motion changes radically with time, during some 
short periods, the central eye of the flow roll changes its shape and its position. During other instants the motion has such strength (high momentum transfer rate) that the jet impacts strongly the narrow faces inducing high velocities in the mould corner (see Fig. 11(b)).

- Since the full throughput is liberated through only two ports the level of turbulence of the discharging jets leads to large velocity fluctuations at the ports. This instability is then inherited in the meniscus where high wave amplitudes are observed as mentioned in the previous section.

- Fluid flow using SEN 2 (Figs. 11(d)-11(f)) maintains a relatively constant flow pattern with time, i.e., velocity fluctuations are smaller than the case of SEN 2 due to a larger number of discharging ports. Flow pattern is a double roll one.

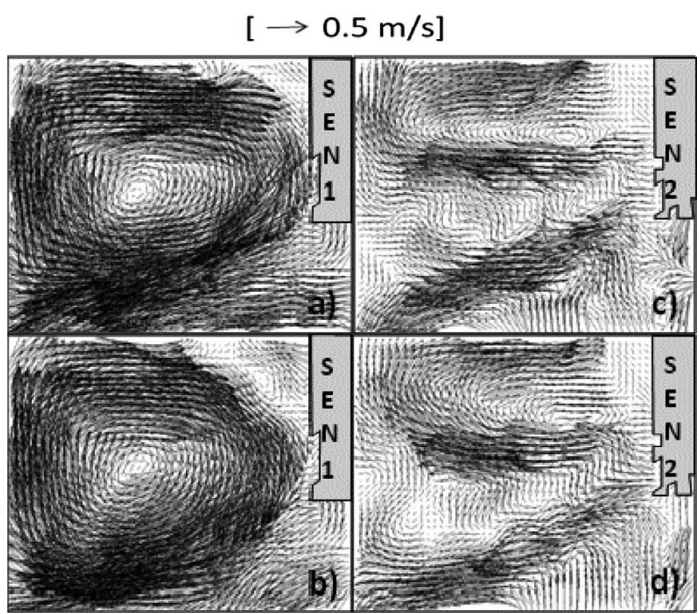

Fig. 12. Velocity fields in the central plane of the mould using $a-b)$ SEN 1 and c-d) SEN 2 at a casting speed of $3.68 \mathrm{~m} / \mathrm{min}$. Time difference among each image is $0.25 \mathrm{~s}$.

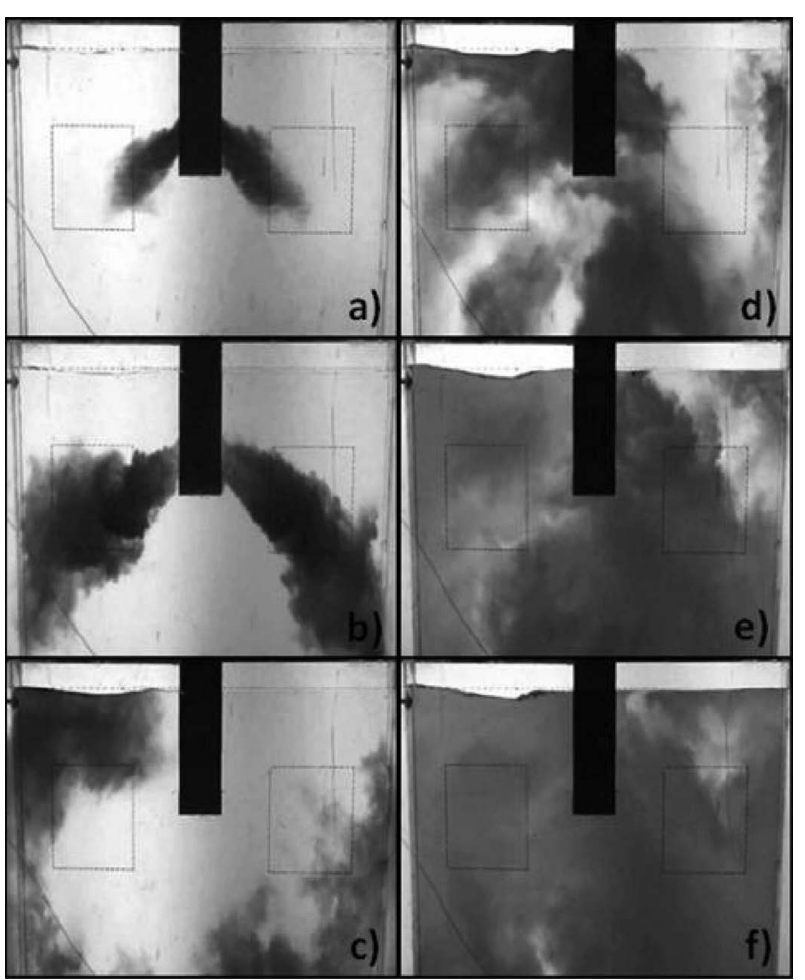

Fig. 13. Flow patterns at different times using SEN 1 at a casting speed of $3.68 \mathrm{~m} / \mathrm{min}$. Times after dye injection are a) $1 \mathrm{~s}$. b) 2 s. c) 3 s. d) 5 s. e) 7 s. f) 9 s.
Using SEN 1 and increasing the casting rate to 3.68 $\mathrm{m} /$ minute, makes the flow pattern less predictable and irregular leaving stagnant regions in the mould corners as is seen in Figs. 12(a) and 12(b) recorded at different times. However, using SEN 2, as is seen in the velocity fields in Figs. 12(c) and 12(d), the flow pattern remains constant with time observing a well defined upper and lower flow rolls. These effects are also evidenced through injection of red dye tracer.

Figures 13(a)-13(f) show the highly irregular, instable and heterogeneous mixing pattern of the tracer using SEN 1 proving the existence of a permanent unstable flow pattern. The rolling effect of the fluid drives the tracer to a very asymmetric distribution and visual observations allowed the identification of intermittent vortexes on the bath surface. At the smaller casting speed this effect is weaker and the mixing pattern tends to be more symmetric. On the other hand, the same experiment performed using SEN 2, Figs. 14(a)$14(f)$, yields a more symmetric distribution of the concentration of the tracer. Due to the specific design of SEN 2 a double roll flow pattern is always attained providing steady operation conditions.

Figures 15(a)-15(b) show the vorticity fields corresponding to Figs. 12(a) and 12(b) for SEN 1 at a casting speed of $3.68 \mathrm{~m} /$ minute. Meanwhile Figs. 15(c) and 15(d) correspond to Figs. 12(c) and 12(d) using SEN 2 at the same casting speed. In the first case it is observed that high flow vorticity is located near the meniscus region, which explains the vortex flows visually observed on the bath surface. Although not shown here this vorticity pattern persists along the time but with changing magnitudes that depend on the instantaneous level of turbulence. In the second case, Figs. 15(c)15(d), smaller vorticity magnitudes near the meniscus regions are observed explaining, then, the higher stability of the flow even at high casting speeds. In order to provide a general visualization of the flows using either of these SEN's the averaged streamlines for a casting speed of 3.68 $\mathrm{m} /$ minute are presented in Figs. 16(a) and 16(b) (corresponding to the velocity fields in Figs. 12(a) and 12(b)) for

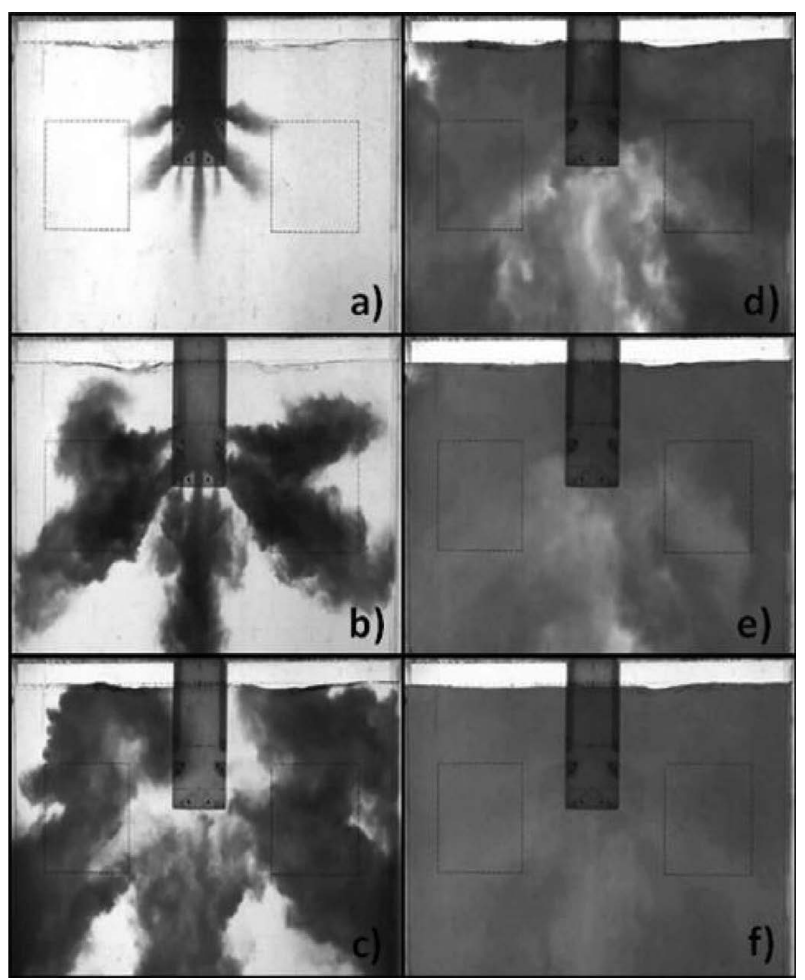

Fig. 14. Flow patterns at different times using SEN 2 at a casting speed of $3.68 \mathrm{~m} / \mathrm{min}$. Times after dye injection are a) $1 \mathrm{~s}$. b) 2 s. c) 3 s. d) 5 s. e) 7 s. f) 9 s. 
SEN 1 and 16(c)-16(d) for SEN 2 (corresponding to the velocity fields in Figs. 12(c) and 12(d)). Figures 16(a) and 16(b) show the high volumetric flows concentrated in the
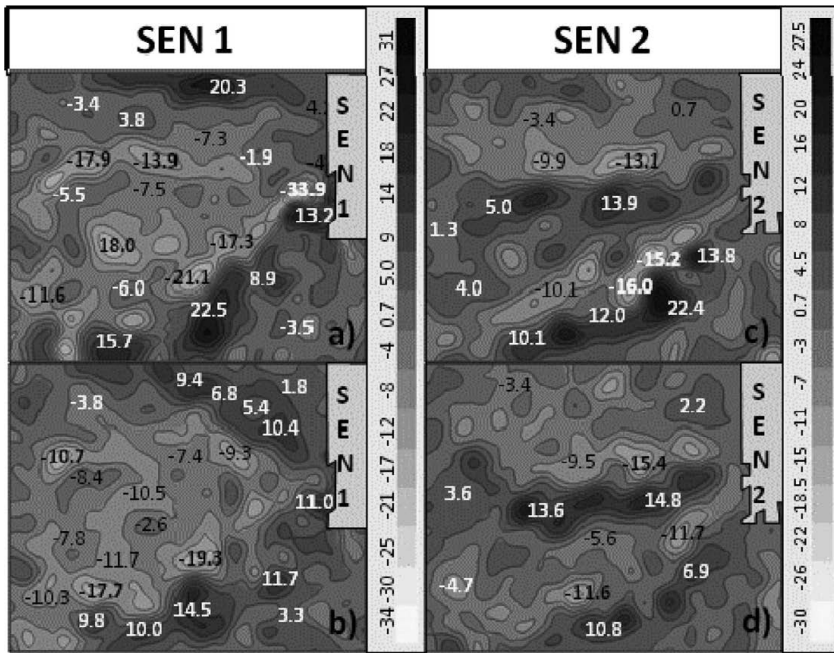

Fig. 15. Vorticity fields $\left(\mathrm{s}^{-1}\right)$ corresponding to the velocity fields a-b) SEN 1 and c-d) SEN 2.

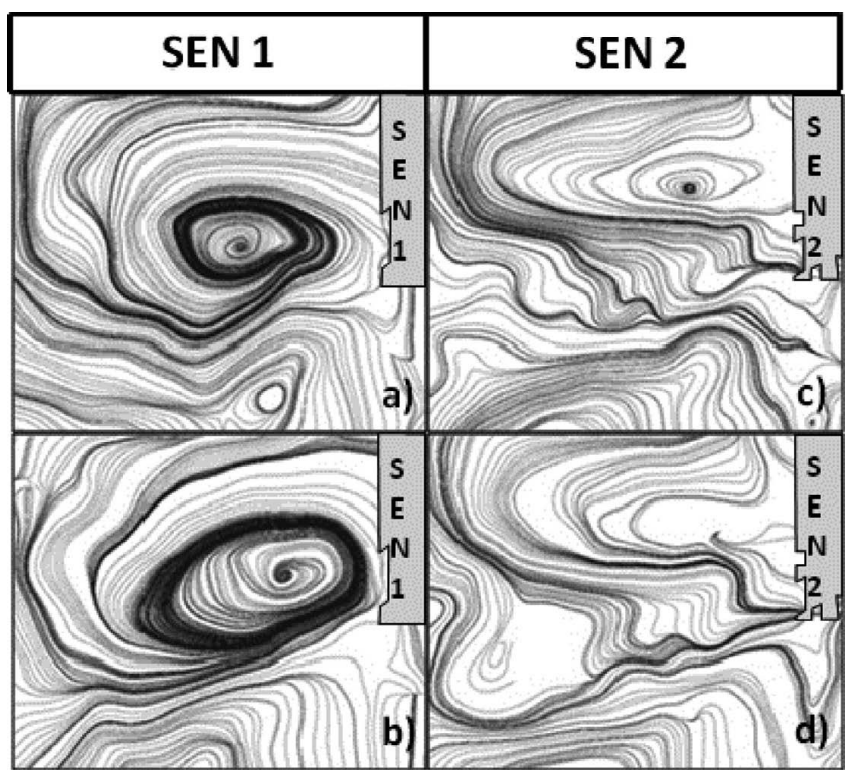

Fig. 16. Streamlines corresponding to the velocity fields $a-b)$ SEN 1 and c-d) SEN 2

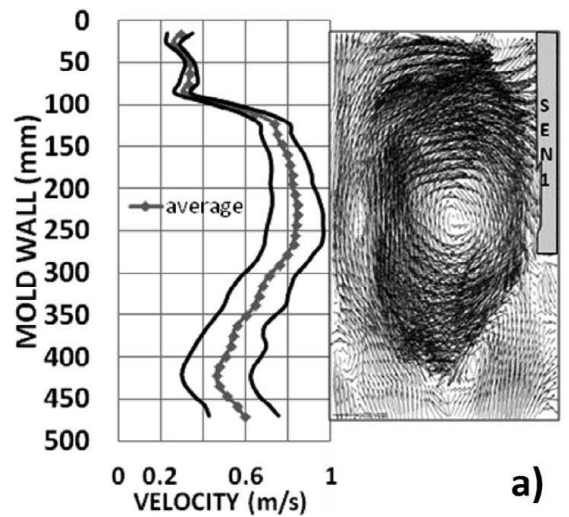

upper flow roll promoted by the high momentum transfer rate of the discharging jet. This condition is responsible of the high mass flux of tracer directed toward the upper part of the mould and is particularly observed in Figs. 13(a) 13(c) where the tracer is rapidly transported by the long roll toward the bath surface. Streamlines in the lower part of the mould are more open indicating smaller fluid flow rates. Different to the this description, SEN 2 in Figs. 16(c) and 16(d), provides streamlines with a more regular separation pattern meaning a more even distribution of fluid flow rates in the whole flow field. Even though the throughput is large the turbulence level is maintained at a level good enough to have a stable meniscus.

Another aspect to be considered is the order of magnitudes corresponding to melt speeds near the shell in the narrow mould face due to the possible "shell washing" effects by convective currents. Figures 17(a) and 17(b) show plots of velocity magnitudes along the mould length at a casting speed of $3.30 \mathrm{~m} / \mathrm{min}$ (along a line which is separated $15 \mathrm{~mm}$ from the mould narrow face in the central plane) from the meniscus level $500 \mathrm{~mm}$ down the mould. These figures include the minimum and maximum velocity magnitudes (obtained from 300 images of velocity fields recorded during the PIV experiments) for SEN 1 and SEN 2, respectively. As can be seen, these magnitudes can be compared with the corresponding images of velocity fields attached to each plot. In this regard, SEN 1 yields maximum fluid velocities of around $1 \mathrm{~m} / \mathrm{s}$ which is a speed high enough to transport thermal energy to induce the shell "washing effect" which may lead to an inevitable strand breakout as has been reported somewhere else. ${ }^{14)}$ However, the corresponding velocity profile yielded by SEN 2 does not surpass the magnitude of $0.4 \mathrm{~m} / \mathrm{s}$ with very small standard deviations. Furthermore, the small variations of fluid velocity ensure stable shell growth under the flow conditions provided by SEN 2 .

\subsection{Flux Entrainment or Entrapment Mechanisms}

Results of water modeling like the ones of the present work can be used to predict the mechanisms of flux entrainment responsible for the generation of slivers and pencil pipes in steel sheets and plates. All possible mechanisms, so far reported in the open literature, ${ }^{15-32)}$ are shown in Figs. 18(a)-18(h). These mechanisms are summarized as follows:

a) Meniscus level fluctuations. Excessive meniscus fluctuations lead to slag entrapment by the dendrites in the solidification front. Factors that enhance this mechanism are increasing casting speed, increases of SEN bore diameter, making more upwards port angles, decreasing SEN immersion depth, decreasing mould width and increasing argon flow rate.

b) Top surface balding. High meniscus speeds push the slag

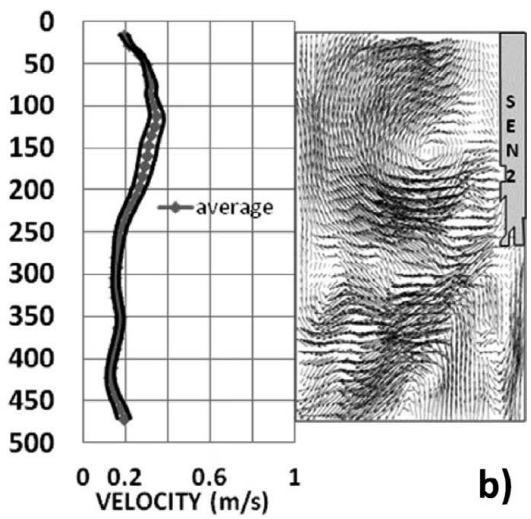

Fig. 17. Velocity profiles along the mould length from the lower side to the mould corner at a casting speed of $3.30 \mathrm{~m} / \mathrm{min}$ a) SEN 1 b) SEN 2 . 
layer including the powder leaving the bath surface exposed to the atmospheric air promoting steel reoxidation and precipitation of alumina.

c) Shear layer instability. Relative motion between steel bath and molten slag can lead to interface instability promoting slag entrainment into the molten steel. The most probable location for this mechanism is just the depressions of the bath surface where steel velocity can reach high magnitudes.

d) Upward flow impingement on meniscus. Generation of and upward spout along narrow faces resulting from a
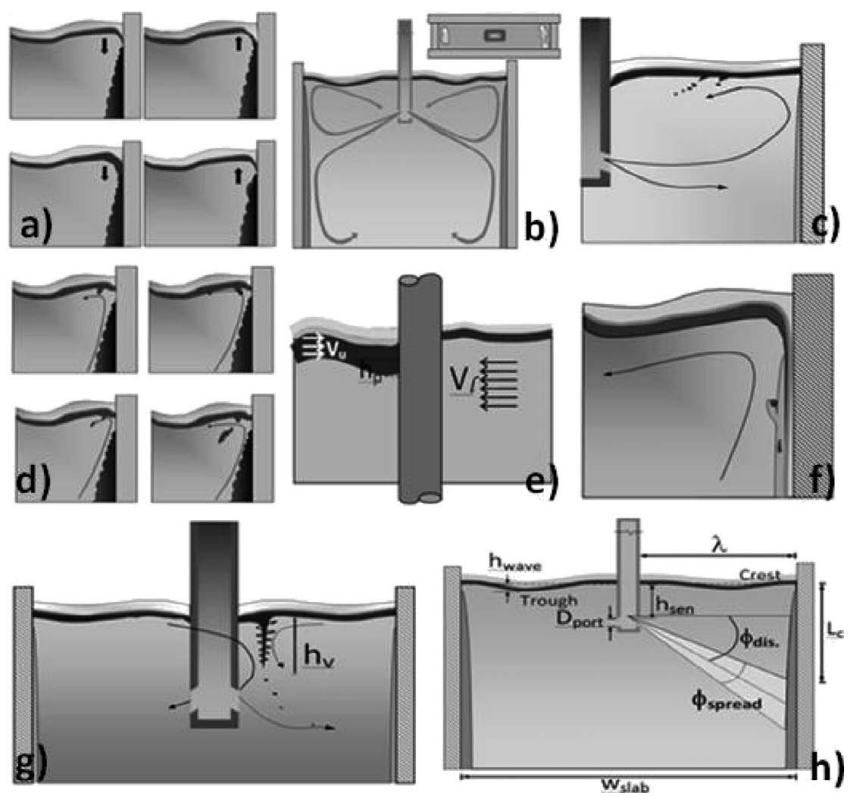

Fig. 18. Flux entrapment mechanisms. a) Meniscus level fluctuations. b) Tops surface balding. c) Shear layer instability. d) Upward flow impinging. e) Slag crawling. f) Meniscus feeding and hook formation. g) Von Karman vortex. h) Meniscus standing wave. high speed double roll flow pattern. This causes slag entrainment by cutting and dragging effects on slag layer. This mechanism is enhanced with thick slag layers and low interfacial tensions.

e) Slag crawling. Generation of excessive gradient pressure at both sides of the SEN inducing an elevation of the surface in the high pressure side. This mechanism is enhanced in asymmetric flows since the high pressure side may smear the slag on the SEN surface. The slag flows downwards reaching the ports and the jet drives it into the melt bulk. Two important parameters of this mechanisms are the constants $\mathrm{C}_{\mathrm{pmax}}$ and $\mathrm{C}_{\mathrm{pmin}}$ given by the expressions,

$$
\begin{aligned}
& C_{p \max }=1.376-0.652 \operatorname{Ln} a \\
& C_{p \min }=1.978-1.065 \operatorname{Ln} a
\end{aligned}
$$

Where $\mathrm{a}$ is the ratio of the major diameter (parallel to the flow direction) to the minor diameter (increasing this aspect ratio lowers the penetration depth) of the discharging ports.

f) Meniscus freezing and hook formation. Hook formation is originated by freezing of the meniscus, due to insufficient heat delivered to the meniscus region near the narrow face. If the hook extends long enough into the melt it can capture rising bubbles and inclusions. Overflowing the frozen meniscus may lead to slag entrapment by meniscus hooks. Ultra Low Carbon steels are more prone to this type of defect.

g) Von Kárman vortex formation. This mechanism is assisted by asymmetric flows which cause uneven flows in the spaces between the broad face and the SEN wall inducing vortices. Slag can be entrapped by vortices and eventually can be trapped by the growing dendrites along the mould broad faces forming slivers defects in the final product.

h) Meniscus standing wave instability. Sub-meniscus melt velocities can be so high that will create surface waves which can turn over to entrain the surface promoting slag entrapment. An important parameter of this mech-

\begin{tabular}{|c|c|c|c|}
\hline Description & Parameters and Equations & Critical Magnitudes & Reference \\
\hline $\begin{array}{l}\text { Fig. 18(a). Meniscus Level Fluctuations. Some } \\
\text { fluctuations of the meniscus can entrain slag } \\
\text { by exposing the dendritic interface of } \\
\text { the top of the solidifying steel shell. }\end{array}$ & $\begin{array}{l}\text { Casting Speed, SEN bore diameter, SEN port angles, } \\
\text { immersion depth, slab width, flow patterns. }\end{array}$ & - & 15 \\
\hline $\begin{array}{l}\text { Fig. 18(b). Top Surface Balding. Uncovering } \\
\text { the melt by excessive velocities at meniscus level. }\end{array}$ & $\begin{array}{l}\text { Flow rates, slag layer thickness, standing wave height, } \\
\text { excessive argon flow rates. }\end{array}$ & - & 16,17 \\
\hline $\begin{array}{l}\text { Fig. 18(c). Shear Layer Instability. Differences } \\
\text { of velocities between slag layer and melt. }\end{array}$ & $\Delta V_{c r i t}=\sqrt[4]{4 g \times\left(\rho_{l}-\rho_{u}\right) \times \Gamma_{u, l} \times\left[\rho_{u}^{-1}+\rho_{l}^{-1}\right]^{2}}$ & $0.38 \mathrm{~m} / \mathrm{s}$ & 18,19 \\
\hline $\begin{array}{l}\text { Fig. 18(d). Upward Flow Impinging on } \\
\text { Meniscus. Upward spout along the narrow } \\
\text { mould faces. }\end{array}$ & $V_{c r i t}=3.065 \times\left(\frac{\Gamma_{u, l}^{0.292} \times g^{0.115}}{\delta^{0.365}}\right) \times\left[\frac{\left(\rho_{l}-\rho_{u}\right)^{0.215}}{\rho_{u}^{0.694}}\right] \times\left(\frac{\mu_{u}^{0.231}}{\mu_{l}^{0.043}}\right)$ & $0.38 \mathrm{~m} / \mathrm{s}$ & 20 \\
\hline $\begin{array}{l}\text { Fig. } 18(\mathrm{e}) \text {. Slag Crawling. Asymmetric buildup } \\
\text { of pressure at both sides of the SEN. }\end{array}$ & $h_{p}=1.9 \frac{C_{p \max } \rho_{u} V_{u}^{2}+C_{p \min } \rho_{l} V_{u}^{2}}{g\left(\rho_{l}-\rho_{u}\right)}$ & $0.035 \mathrm{~m}$ & 21,22 \\
\hline $\begin{array}{l}\text { Fig. 18(f). Meniscus Freezing and Hook } \\
\text { Formation. Slag entrapment at shell hooks. }\end{array}$ & Flow velocity, superheat, SEN port angles. & - & 23,24 \\
\hline $\begin{array}{l}\text { Fig. } 18(\mathrm{~g}) \text {. Von Kármán Vortex. Periodic } \\
\text { shedding of vortices near the SEN walls. }\end{array}$ & $h_{v}=\left(\frac{V_{m c}^{2}}{g}\right)\left(\frac{\rho_{l}}{\rho_{l}-\rho_{u}}\right)+c\left(\frac{\Delta V_{s}^{2} \times \rho_{u}}{g \times\left(\rho_{l}-\rho_{u}\right)}\right)^{0.55}$ & $0.060 \mathrm{~m}$ & $25-29$ \\
\hline Fig. 18(h). Meniscus Standing Wave. & $h_{\text {wave }}=0.31 \times\left(\frac{V_{\text {port }}^{2}}{g}\right) \times\left(\frac{D_{\text {port }}}{L}\right) \times\left(\frac{\rho_{l}+\rho_{u}}{\rho_{l}-\rho_{u}}\right)$ & $0.015 \mathrm{~m}$ & 30,31 \\
\hline
\end{tabular}

Table 2. Summary of mould entrapment mechanisms. 
anism is the critical ratio between the wave height and the wave length of the bath oscillation given as,

$$
\left(\frac{h_{\text {wave }}}{\lambda}\right)_{\text {crit }}=0.21+0.14\left(\frac{\rho_{u}}{\rho_{l}}\right)^{2}
$$

Where $\lambda$ is the distance between the SEN and the narrow mould face, $\rho_{u}$ and $\rho_{l}$ are densities of the upper phase (slag) and lower phase (steel), respectively. Table 2 summarizes these mechanisms including mathematical expressions and main parameters to be able to calculate the critical magnitudes of flow variables that may lead to slag entrainment or entrapment by any of the mechanisms mentioned above. The third column of Table 2 shows the magnitudes of the critical variables from which the slag entrainment or entrapment mechanisms start to operate.

Various flow variables were used to calculate the critical parameters that may indicate the predominance of one mechanism over the other such as impinging jet distance from meniscus, jet angle, meniscus speeds, etc., all obtained from the tracer injection images and PIV measurements. Other variables like densities, surface tensions and viscosities of steel and slag were consulted in Ref. 33). Table 3 shows these physical parameters; the interfacial tension was calculated through the magnitudes of surface tensions of each phase and calculated through the Garifalco's expression: ${ }^{34)}$

$$
\gamma_{m s}=\gamma_{m}+\gamma_{s}-\phi\left(\gamma_{m} \gamma_{s}\right)^{0.5}
$$

Where $\phi-W_{C}^{m s} / W_{C}^{M} W_{C}^{a}$ and $W_{C}^{m}, W_{C}^{S}$ are the works of cohesion of metal and slag phase and $W_{A}^{m s}$ is the work of adhesion at the metal-slag interface.

The results of these calculations are presented in Table 4 and they are compared with the corresponding critical magnitudes for each operating parameter reported in the last column. Calculated data for each SEN show minimum and maximum magnitudes of those parameters according to the statistical standard deviations of velocity fields measured by

Table 3. Physical chemical properties of phases.

\begin{tabular}{lcc}
\hline \multicolumn{1}{c}{ Properties } & Steel (1873 K) & Slag $(1873 \mathrm{~K})$ \\
\hline Density & $7014 \mathrm{~kg} / \mathrm{m}^{3}$ & $2600 \mathrm{~kg} / \mathrm{m}^{3}$ \\
Surface Tension & $1.16 \mathrm{~N} / \mathrm{m}$ & $0.524 \mathrm{~N} / \mathrm{m}$ \\
Interfacial Tension (metal-slag) & \multicolumn{2}{c}{$0.46 \mathrm{~N} / \mathrm{m}$} \\
Molecular Viscosity & $0.0064 \mathrm{~Pa}-\mathrm{s}$ & $0.2 \mathrm{~Pa}-\mathrm{s}$ \\
Layer thickness of liquid slag & - & $0.01 \mathrm{~m}$ \\
\hline
\end{tabular}

the PIV equipment and an averaged magnitude was taken from 300 images of the velocity fields in the PIV files. As is seen in this table, most of the conditions exceed the critical magnitudes using SEN 1, indicated by the black and enlarged numerical data. This is particularly true when these parameters are taken from the averaged velocity fields and from the maximum magnitudes derived from the standard deviations. Even, in some cases when the minimum magnitudes of velocities where considered in these calculations SEN 1 exceeds the critical magnitudes. Therefore, it can be concluded that all flux entrainment mechanisms may act using SEN 1 at both casting speeds. And very probably mechanisms (a), (b) and (f) are also acting under these particular conditions. Given the turbulence of the flow the severity of flux entrapment can be certainly overwhelming making very clear the high level of turbulence provided by SEN 1. Meanwhile, SEN 2 yields only few cases where flux entrainment can be expected, it is apparent that meniscus standing wave mechanism can actually work at average and maximum velocities.

\section{Conclusions}

Using a two-port SEN in a medium thickness slab mould induces very high levels of turbulence during steel feeding operations. For that purpose a full scale water model was built and steel flow characterization was performed using various techniques: Level sensors of meniscus levels, PIV measurements and tracer injection experiments. Through the results of this characterization a 7-port SEN was designed, which demonstrates good performance to control flow turbulence and meniscus stability. The conclusions derived from the results obtained in this study are as follows here:

- Flow turbulence using the original SEN 1 is very intensive and promotes large amplitude waves at meniscus level.

- Flow turbulence is evidenced by large fluid velocities in the sub-meniscus region and large field areas with negative vorticity magnitudes. The flow pattern can be denominated as permanent unstable (not double roll neither single flow).

- Uncoupled oscillations of meniscus level at both sides of the current SEN indicate the existence of slag crawling mechanism for slag entrainment. Other mechanisms are Von Karman vortex, upward impinging and other complex mechanisms. These conclusions were derived directly from PIV measurements and empirical expressions designed for each slag entrainment mechanism.

- The proposed SEN eliminates all fluid flow flaws iden-

\begin{tabular}{|c|c|c|c|c|c|c|c|c|c|}
\hline \multirow{2}{*}{ Mechanisms } & \multirow{2}{*}{$\begin{array}{l}\text { Casting Speed } \\
\qquad(\mathrm{m} / \mathrm{min})\end{array}$} & \multirow{2}{*}{$\begin{array}{c}\text { Location Speed } \\
\text { Mesurements by PIV }\end{array}$} & \multicolumn{3}{|c|}{ SEN 1} & \multicolumn{3}{|c|}{ SEN 2} & \multirow{2}{*}{$\begin{array}{c}\text { Critical } \\
\text { Parameters }\end{array}$} \\
\hline & & & Min. & Ave. & Max. & Min. & Ave. & Max. & \\
\hline $\begin{array}{l}\text { c) Shear Layer } \\
\text { Instability }\end{array}$ & 3.30 & $\begin{array}{l}\text { At } 1 / 4 \text { of the mould width } \\
\text { near bath surface. }\end{array}$ & 0.85 & 1.09 & 1.34 & 0.25 & 0.40 & 0.55 & $0.386 \mathrm{~m} / \mathrm{s}$ \\
\hline \multirow{2}{*}{$\begin{array}{l}\text { d) Upward } \\
\text { Flow Impinging }\end{array}$} & 3.30 & \multirow{2}{*}{$\begin{array}{l}\text { On the corner near } \\
\text { to the narrow face. }\end{array}$} & 0.312 & 0.492 & 0.674 & 0.204 & 0.269 & 0.354 & \multirow{2}{*}{$0.380 \mathrm{~m} / \mathrm{s}$} \\
\hline & 3.68 & & 0.260 & 0.426 & 0.595 & 0.178 & 0.247 & 0.329 & \\
\hline e) Slag Crawling & 3.30 & $\begin{array}{l}\text { Near to the wall of SEN, } \\
\text { at meniscus level. }\end{array}$ & 0.007 & 0.082 & 0.247 & 0.001 & 0.016 & 0.052 & $0.035 \mathrm{~m}$ \\
\hline \multirow{2}{*}{$\begin{array}{l}\text { g) Von Kármán } \\
\text { Vortex }\end{array}$} & 3.30 & \multirow{2}{*}{$\begin{array}{l}\text { Vertical and horizontal } \\
\text { velocities at } 1 / 4 \text { of mould } \\
\text { width, along SEN length }\end{array}$} & 0.116 & 0.193 & 0.289 & 0.010 & 0.022 & 0.039 & \multirow{2}{*}{$0.060 \mathrm{~m}$} \\
\hline & 3.68 & & 0.043 & 0.133 & 0.271 & 0.014 & 0.033 & 0.050 & \\
\hline \multirow{2}{*}{$\begin{array}{l}\text { h) Meniscus } \\
\text { Standing Wave }\end{array}$} & 3.30 & \multirow{2}{*}{ Velocity in the jet core. } & 0.023 & 0.043 & 0.069 & 0.006 & 0.014 & 0.022 & \multirow{2}{*}{$0.015 \mathrm{~m}$} \\
\hline & 3.68 & & 0.027 & 0.044 & 0.061 & 0.009 & 0.018 & 0.028 & \\
\hline
\end{tabular}

Table 4. Summary of mould entrapment mechanisms acting in the mould. 
tified when the current SEN is used and distributes better the mass flow in the whole fluid volume in the mould. This effect stabilizes the meniscus level sending fluid to the mould corner in order to avoid excessive shell growth and possible hook cracks. The new SEN design provides a permanent double roll flow pattern.

- The experimental results indicate that by using SEN 2 is possible to obtain a velocity profile along the mould length, near the narrow mould face, with near constant and smaller magnitudes ensuring a possible consistent shell growth in the actual caster.

\section{Acknowledgements}

YSGM acknowledges the scholarship provided by The National Council of Science and Technology of Mexico to carryout her MSc. studies at IPN and to the PIFI system for financial support. RDM gives the thanks to the institutions IPN, SNI, COFAA and CoNaCyT for their permanent support to the Process Engineering Group.

\section{Nomenclature}

V: velocity

$\mathrm{g}$ : acceleration due to gravity

$h_{p}$ : the penetration depth that the upper fluid crawls down the cylinder

$\mathrm{V}_{\mathrm{u}}$ : slag velocity

$\mathrm{V}_{1}$ : steel velocity

$\mathrm{h}_{\mathrm{v}}$ : vortex depth

$\mathrm{V}_{\mathrm{mc}}$ : horizontal steel velocity in the center of the mould and $10 \mathrm{~mm}$ below the water-oil interface and halfway between the SEN and wide face

$\Delta \mathrm{V}_{\mathrm{s}}$ : Vertical velocity measure $50 \mathrm{~mm}$ below the steelslag interface $\mathrm{C}=0.0562$ is a constant

$\mathrm{h}_{\text {wave: }}$ wave height, defined as the vertical distance between the lowest point and highest point of the surface level.

$\mathrm{W}_{\text {slab: }}$ : width of the slab

$\mathrm{L}_{\mathrm{c}}$ : distance from the meniscus to the impinging point of the jet on the narrow face

$\mathrm{V}_{\text {port }}$ : velocity of the molten steel at the SEN ports

$\mathrm{D}_{\text {port }}$ : diameter of the SEN port

$\mathrm{W}_{\mathrm{A}}$ : adhesion work

$\mathrm{W}_{\mathrm{C}}$ : cohesion work

\section{Greek letters}

$\lambda$ : wavelength, the distance between the outer SEN wall and the narrow faces.

$\Phi_{\text {discharge: }}$ discharge angle of the jet

$\Phi_{\text {spread: }}$ spread angle of the jet

$\mu$ : molecular viscosity $\rho:$ density

$\Gamma_{\mathrm{u}, 1}$ : interfacial tension between slag and steel

$\Gamma_{\mathrm{s}}$ : surface tension of slag

$\Gamma_{\mathrm{m}}$ : surface tension of metal

\section{REFERENCES}

1) M. Suzuki, M. Suzuki and M. Nakada: ISIJ Int., 41 (2001), 670.

2) W. H. Emling and T. A. Waugaman: Steelmaking Conf. Proc., ISS, Warrendale, PA, (1994), 371.

3) R. M. McDavid and B. G. Thomas: Metall. Mater. Trans. B, 27 (1996), 672.

4) Q. He, G. Evans, R. Serje and T. Jaques: Proc. of AISTech 2009, AIST, Warrendale, PA, (2009), 573.

5) A. Ramos-Banderas, R. Sanchez-Perez and R. D. Morales: Metall. Mater. Trans. B, 35B (2004), 449.

6) D. Gupta, S. Chakraborty and K. Lahiri: ISIJ Int., 37 (1997), 654.

7) T. Honeyands and J. Herbertson: Steel Res., 66 (1995), 287.

8) E. Torres-Alonso, R. D. Morales, L. G. Demedices, A. Nájera, J. Palafox-Ramos and P. Ramirez-Lopez: ISIJ Int., 47 (2007), 679.

9) E. Torres Alonso, R. D. Morales, S. Garcia-Hernandez and J. PalafoxRamos: Metall. Mater. Trans. B, 41B (2010), 583.

10) E. Torres-Alonso, R. D. Morales and S. Garcia-Hernandez: Metall. Mater. Trans. B, 41B (2010), 675.

11) R. D. Morales, J. Palafox-Ramos, L. Garcia-Demedices and R. Sanchez-Perez: ISIJ Int., 44 (2004), 1384.

12) R. D. Morales, Y. Tang, G. Nitzl, C. Eglsaeer and G. Hackl: ISIJ Int., 52 (2012), 1607.

13) R. Sánchez-Pérez, R. D. Morales, M. Díaz-Cruz and O. Olivares: ISIJ Int., 43 (2003), 637.

14) A. Najera Bastida, R. D. Morales, S. Garcia-Hernandez, E. TorresAlonso and A. Espino-Zarate: ISIJ Int., 50 (2010), 830.

15) T. Teshima, M. Osame, K. Okimoto and Y. Nimura: 71st Steelmaking Conf. Proc., ISS, Warrendale, PA, (1988), 111.

16) J. Kubota. K. Okimoto, A. Shirayama and H. Murakami: 74th Steelmaking Conf. Proc., ISS, Warrendale, PA, (1988), 233.

17) D. Gupta and A. K. Lahiri: Metall. Mater. Trans. B, 27B (2004), 695.

18) D. Gupta and A. K. Lahiri: Ironmaking Steelmaking, 23 (1996), 361.

19) M. Iguchi, J. Yoshida, T. Shimizu and Y. Mizuno: ISIJ Int., 40 (2000), 685.

20) P. Cha and J. Yoon: Metall. Mater. Trans. B, 31B (2000), 317.

21) J. M. Harman and A. W. Cramb: 79th Steelmaking Conf., ISS, Warrendale, PA, (1996), 773.

22) J. Yoshida, T. Ohmi and M. Iguchi: ISIJ Int., 44 (2005), 1160.

23) Y. Ueda, T. Kida and M. Iguchi: ISIJ Int., 44 (2005), 1403.

24) J. Segupta, B. G. Thomas and H. J. Kim: Metall. Meter. Trans. A, 37A (2006), 1597.

25) G. Lee, B. G. Thomas and S. Kim: Acta Mater., 55 (2007), 6705.

26) B. Li and G. Tsukihashi: ISIJ Int., 45 (2005), 30.

27) N. Kasai and M. Iguchi: ISIJ Int., 47 (2007), 982.

28) Q. He: ISIJ Int., 33 (1993), 343.

29) M. Gebhard, Q. He and J. Herberson: 76th Steelmaking Conf. Proc., Iron and Steel Society, Warrendale PA, (1993), 441.

30) B. Li and F. Tsukihashi: ISIJ Int., 46 (2006), 404.

31) J. W. Rottman: J. Fluid Mech., 124 (1982), 283.

32) A. Theodorakakos and G. Bergeles: Metall. Meter. Trans. B, 29B (1998), 1117.

33) Slag Atlas, 2nd edition, ed. by Verein Deutscher Eisenhuttenleute (VDEh), Verlag Stahleisen, Düsseldorf, Germany, (2008), 342.

34) K. C. Mills and S. Sridhar: Belton Symp. Proc., Iron and Steel Soc., Warrendale, PA, (2000), 211. 